OPEN ACCESS

Edited by:

Bingru Huang,

Rutgers University, United States

Reviewed by:

Hong Luo,

Clemson University, United States

Xiqing $\mathrm{Ma}$,

China Agricultural University, China

${ }^{*}$ Correspondence:

Yi Li

yi.li@uconn.edu

Tingting Gu

gutingting@njau.edu.cn

tThese authors have contributed equally to this work.

Specialty section:

This article was submitted to

Crop Science and Horticulture,

a section of the journal

Frontiers in Plant Science

Received: 21 March 2017 Accepted: 09 May 2017

Published: 26 May 2017

Citation:

Li W, Katin-Grazzini L, Gu X, Wang $X$, El-Tanbouly $R$, Yer $H$,

Thammina $C$, Inguagiato $J$, Guillard K, McAvoy RJ, Wegrzyn J, Gu T and Li Y (2017) Transcriptome Analysis Reveals Differential Gene Expression and a Possible Role of Gibberellins in a Shade-Tolerant Mutant of Perennial Ryegrass.

Front. Plant Sci. 8:868. doi: 10.3389/fpls.2017.00868

\section{Transcriptome Analysis Reveals Differential Gene Expression and a Possible Role of Gibberellins in a Shade-Tolerant Mutant of Perennial Ryegrass}

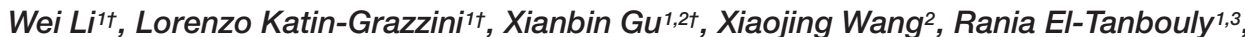
Huseyin Yer', Chandra Thammina', John Inguagiato ${ }^{1,}$ Karl Guillard $^{1}$, Richard J. McAvoy ${ }^{1}$, Jill Wegrzyn ${ }^{4}$, Tingting $\mathrm{Gu}^{2 *}$ and Yi Li ${ }^{1,2 *}$

'Department of Plant Science and Landscape Architecture, University of Connecticut, Storrs, CT, United States, ${ }^{2}$ College of Horticulture and State Key Laboratory of Crop Genetics and Germplasm Enhancement, Nanjing Agricultural University, Nanjing, China, ${ }^{3}$ Department of Floriculture, Ornamental, Horticulture and Landscape Gardening, Faculty of Agriculture, Alexandria University, Alexandria, Egypt, ${ }^{4}$ Department of Ecology and Evolutionary Biology, University of Connecticut, Storrs, CT, United States

The molecular basis behind shade tolerance in plants is not fully understood. Previously, we have shown that a connection may exist between shade tolerance and dwarfism, however, the mechanism connecting these phenotypes is not well understood. In order to clarify this connection, we analyzed the transcriptome of a previously identified shade-tolerant mutant of perennial ryegrass (Lolium perenne L.) called shadow-1. shadow-1 mutant plants are dwarf, and are significantly tolerant to shade in a number of environments compared to wild-type controls. In this study, we treated shadow-1 and wild-type plants with 95\% shade for 2 weeks and compared the transcriptomes of these shade-treated individuals with both genotypes exposed to full light. We identified 2,200 differentially expressed genes (DEGs) (1,096 up-regulated and 1,104 down-regulated) in shadow-1 mutants, compared to wild type, following exposure to shade stress. Of these DEGs, 329 were unique to shadow-1 plants kept under shade and were not found in any other comparisons that we made. We found 2,245 DEGs (1,153 up-regulated and 1,092 down-regulated) in shadow-1 plants, compared to wild-type, under light, with 485 DEGs unique to shadow-1 plants under light. We examined the expression of gibberellin (GA) biosynthesis genes and found that they were down-regulated in shadow-1 plants compared to wild type, notably gibberellin 20 oxidase (GA20ox), which was downregulated to $3.3 \%$ (96.7\% reduction) of the wild-type expression level under shade conditions. One GA response gene, lipid transfer protein 3 (LTP3), was also downregulated to $41.5 \%$ in shadow-1 plants under shade conditions when compared to the expression level in the wild type. These data provide valuable insight into a role that GA plays in dwarfism and shade tolerance, as exemplified by shadow-1 plants, and could serve as a guide for plant breeders interested in developing new cultivars with either of these traits.

Keywords: shadow-1, transcriptome analysis, differentially expressed genes, dwarfism, gibberellins, shade tolerance 


\section{INTRODUCTION}

Perennial ryegrass (Lolium perenne L.) is one of the most widely cultivated cool-season turfgrass species in the world (Jiang and Huang, 2001; Chen J. et al., 2016). Known for its fast establishment, perennial ryegrass is favored for ornamental use as well as for livestock grazing (Grinberg et al., 2016). While perennial ryegrass is incorporated into many seed mixtures due to its positive traits, it is seldom grown by itself because of its sensitivity to a number of environmental stresses (Gardner and Taylor, 2002; Tegg and Lane, 2004). Perennial ryegrass struggles to grow in overly shady environments, exhibiting shade avoidance response (SAR). This condition is characterized by weak growth, overly elongated leaves, and chlorosis (Franklin and Whitelam, 2005). SAR and other symptoms of shade stress impact virtually all plant taxa, and a high degree of shade has a negative impact on the growth and development of all plants (Nozue et al., 2015). In cereal crop plants, such as maize, shade can inhibit lateral branching, leading to a reduction in overall vegetative biomass (Kebrom et al., 2006; Whipple et al., 2011). Shade has also been shown to reduce the production of grains, such as kernels in maize, as well as seeds, as seen in Brassica rapa (Page et al., 2010; Procko et al., 2014).

In seedlings, shade causes etiolation, which is characterized by elongation of the hypocotyls and petioles and, in some cases, the inhibition of cotyledon expansion and reduction in lateral roots (Procko et al., 2014). Petiole elongation is also a symptom of shade stress in adult plants (Kozuka et al., 2005; Sasidharan et al., 2010). These various shade responses are known to be regulated by light-sensing pigments called phytochromes. Shade conditions reduce the activity of phytochromes, of which phytochromes $\mathrm{A}$ and B (PhyA, PhyB) have been shown to be especially important. Phytochromes are responsible for repressing the DNA binding activity of phytochrome interacting factors (PIFs; Park et al., 2012). Once free of PhyB repression, PIFs are able to activate various shade-associated physiological responses, such as stem and petiole elongation, through their activity as transcription factors (Lorrain et al., 2008). DELLA proteins, through proteinprotein interaction, also repress PIF activity (De Lucas et al., 2008; Feng et al., 2008). DELLA proteins are degraded in the presence of gibberellin (GA) after binding to the GA receptor GID1 via the E3 ubiquitination pathway (Sun, 2008).

In many plant species, shade response is controlled through various phytohormone response pathways, most notably the GA pathway (Yamaguchi, 2008; Colebrook et al., 2014). Previously, we have suggested that GA content has a potential impact on the shade tolerance exhibited by shadow-1 mutant plants (Li et al., 2016). GAs are terpenoid products, and GA biosynthesis begins when geranylgeranyl pyrophosphate $(G G P P)$ is catalyzed into ent-copalyl pyrophosphate by ent-copalyl diphosphate synthase. This product is then modified by a number of upstream biosynthesis enzymes, namely: ent-kaurene synthase $(K S)$, entkaurene oxidase $(K O)$, and ent-kaurenoic acid oxidase (KAO). The final steps of bioactive GA biosynthesis are catalyzed by gibberellin 20 oxidase (GA20ox) and gibberellin 3 oxidase (GA3ox). The process of deactivation of bioactive GAs is governed by gibberellin 2 oxidase (GA2ox; Hedden and Phillips, 2000; Chen S. et al., 2016).

In congested areas, whether with buildings in urban areas or with trees in rural areas, it can be difficult to find growing space with adequate light exposure for ornamental plants (Pons and Poorter, 2014). Understanding the mechanisms behind shade tolerance would make it possible to develop solutions to the challenges of growing plants in low-light environments. shadow-1 is a dwarf, shade-tolerant perennial ryegrass mutant. When subjected to severe shade stress $(95 \%$ light reduction) shadow-1 plants are significantly resistant to SAR (Li et al., 2016). The shadow-1 mutant line represents a valuable opportunity to study the shade response pathway in monocots.

In an attempt to uncover the genetic mechanisms behind dwarfism and shade tolerance in shadow-1 perennial ryegrass, we have treated shadow-1 and wild-type plants with $95 \%$ shade and compared their transcriptomes to plants kept under full light. Through examination of differential gene expression within the GA biosynthesis and response pathways of shadow-1 mutant plants, we have implicated decreases in GA content as a likely mechanism for shade tolerance, as well as dwarfism, in these plants. These results provide some insight into the role that GAs may play in shade response, as well as possible strategies for breeding shade tolerant crop plants.

\section{MATERIALS AND METHODS}

\section{Plant Treatment and Tissue Sampling}

shadow-1 and wild-type plants were vegetatively propagated in rectangular pots $(15 \mathrm{~cm} \times 11 \mathrm{~cm} \times 5 \mathrm{~cm})$. Plant roots and shoots were cut to $2.5 \mathrm{~cm}$ and six groups of two tillers were evenly spread within each pot. Plants were maintained at a $5 \mathrm{~cm}$ height in full light for 6 weeks. Individuals selected for shade-stress treatment were placed in a 95\% shade environment in the greenhouse, which was created by the use of black polyfiber cloth. Those selected for full-sunlight treatment were left out in the open in the greenhouse. After growing for an additional 2 weeks under either light or $95 \%$ shade, leaf tissue was collected from six pots (one biological replicate) for each genotype (wild type or shadow-1) under each treatment (light or shade). A total of three replicates were collected for each genotype under each treatment. Tissue was collected by cutting young leaves directly into a beaker of liquid nitrogen in an effort to preserve mRNA. For shade-treated plants, this was done in a darkroom environment to avoid light contamination.

\section{RNA Extraction and Library Preparation}

Total plant RNA was extracted using the RNeasy Plant Mini Kit, including RNase-Free DNase set (Qiagen, Valencia, CA, United States), according to the manufacturer's protocol. RNA purity and concentration were measured using the NanoDrop 2000 spectrophotometer (Thermo Fisher Scientific, Waltham, MA, United States). To further assess RNA quality, total RNA was analyzed on the Agilent TapeStation 2200 (Agilent Technologies, Santa Clara, CA, United States) using the RNA 
High Sensitivity assay. Ribosomal Integrity Numbers (RINe) were recorded for each sample. Only samples with RINe values above 7.0 were used for library preparation. Total RNA samples were prepared for mRNA-Sequencing using the Illumina TruSeq Stranded mRNA Sample Preparation kit following the manufacturer's protocol (Illumina, San Diego, CA, United States). Libraries were validated for length and adapter dimer removal using the Agilent TapeStation 2200 D1000 High Sensitivity assay (Agilent Technologies, Santa Clara, CA, United States) and were then quantified and normalized using the dsDNA High Sensitivity Assay for Qubit 2.0 (Life Technologies, Carlsbad, CA, United States). Libraries were prepared for the Illumina HiSeq 2500 (v.4 chemistry) in High Output mode $(2 \times 100 \mathrm{bp})$. A total of 12 libraries were sequenced across two lanes.

\section{Differential Expression Analysis and Functional Annotation}

Clean reads were obtained by first removing adapter sequences, and then filtering out reads with over $20 \%$ low-Q-value $(\leq 20)$ bases, as well as reads with more than $5 \%$ ambiguous " $\mathrm{N}$ " bases. The clean reads were then aligned to the perennial ryegrass genome assembled by Byrne et al. (2015) using default parameters in Tophat2 software (Kim et al., 2013). Gene expression levels were calculated as reads per kilobase of transcript per million mapped reads (RPKM). Differentially expressed genes (DEGs) were defined as genes having a false discovery rate (FDR) $\leq 0.05$ and an absolute $\log _{2}$ fold change value $\geq 1$. To further characterize the function of DEGs, they were mapped to Gene Ontology (GO) classifications using Blast2GO (Conesa and Götz, 2008). Three categories of GO annotations were analyzed: biological process, molecular function, and cellular component. To uncover GA biosynthesis genes and GA response gene of perennial ryegrass, BLASTP was performed against the translated perennial ryegrass reference genome for each gene of interest. The top hits with an E-value $<10^{-4}$ were aligned using ClustalX 2.0 (Larkin et al., 2007). A phylogenetic tree was constructed for all selected hits by PHYML version 3.0 using the maximum likelihood method (Guindon et al., 2010) under the JTT evolutionary model. The closest neighbor for each protein was designated as the putative homolog for that protein in perennial ryegrass. A representative phylogenetic tree was provided in the Supplementary File 1.

\section{Quantitative Real-Time PCR Analysis}

Three genes: KS, KAO, and GA20ox were analyzed using quantitative real-time PCR (qRT-PCR). New plant material was harvested, and RNA was extracted, as previously described. The iScript ${ }^{\mathrm{TM}}$ cDNA Synthesis Kit (Bio-Rad Laboratories, Richmond, CA, United States) was used to synthesize cDNA, and cDNA products were utilized for qRT-PCR assays using SsoFast ${ }^{\text {TM }}$ EvaGreen ${ }^{\circledR} \quad$ Supermix (Bio-Rad Laboratories, Richmond, CA, United States) on a CFX96 ${ }^{\mathrm{TM}}$ Real-Time PCR detection system (Bio-Rad Laboratories, Richmond, CA, United States). Primer sequences for all genes analyzed are as follows: $K S$ forward: 5'-GGAAACCTGCTAGACTGGAA-3', $K S$ reverse: $5^{\prime}$-ATTTAGGTACCCGAGGGCTT-3', KAO forward: 5'-CAGGAAGATGGAGTACCTCT-3', KAO reverse: 5'-ATG TGCACAGTCCTGTACCA-3', GA20ox forward: 5'-GACTTCA CGCAGAAGCACTA-3', GA20ox reverse: 5'-GCAGATGCA GAGAAGCAGAA-3', LpGAPDH forward: 5'-CATCACCATTG TCTCCAACG-3', LpGAPDH reverse: 5'-AACCTTCAACGA TGCCAAAC-3'. The native glyceraldehyde-3-phosphate dehydrogenase $(L p G A P D H)$ gene was used as the internal control (Petersen et al., 2004; Kovi et al., 2016). Data were analyzed using CFX Manager ${ }^{\mathrm{TM}}$ software version 2.0. The expression levels in each sample was normalized using the expression level of $L p G A P D H$ gene in the same sample. Three biological replicates were performed with each type of sample. Means of gene expression levels between shadow-1 and wild type were compared using the two-tailed Student's $t$-test with the pooled variance (Steel et al., 1997).

\section{RESULTS}

\section{Sequencing and Mapping of the shadow-1 Transcriptome}

When grown in the greenhouse under full light conditions, shadow-1 plants exhibited dwarfism, categorized by reduced canopy heights compared to wild type (Figure 1A). Following 2 weeks of shade treatment, shadow-1 plants were found to be more tolerant to shade compared to wild type, as evidenced by a significant reduction in leaf elongation and the retention of a healthy, green appearance (Figure 1B). These results are consistent with previously reported analysis of the shadow-1 mutant line (Li et al., 2016).

Following the 2 weeks of shade treatment, leaf tissue samples were harvested from the shadow-1 and wild-type plants, kept under both shade and light conditions, for transcriptome analysis. We used three biological replicates for each genetic background under each treatment. Transcriptome sequencing data were deposited in the NCBI SRA database under the accession number SRP102018. Through sequencing, we generated a total of 657,122,180 raw reads and 633,014,566 clean reads. The average Q20 and Q30 scores for clean reads among all 12 samples were 95.88 and $90.40 \%$, respectively. For these reads, the average GC content was $50.42 \%$ (Table 1). We used the perennial ryegrass genome assembled by Byrne et al. (2015) as a reference against which the clean reads from each sample were mapped. We were able to map around $75 \%$ of the clean reads for each sample group to the reference genome (Table 2).

We have compared gene expression among the three biological replicates for all four sample types: wild type kept under light, shadow-1 kept under light, wild type treated with shade, and shadow-1 treated with shade. The similarity of expression profiles between the replicates was determined by a Pearson correlation coefficient analysis. We found that the three biological replicates for each sample type were highly correlated $(r>0.96)$, demonstrating consistency between replicates regarding to DEGs (Figure 2). 

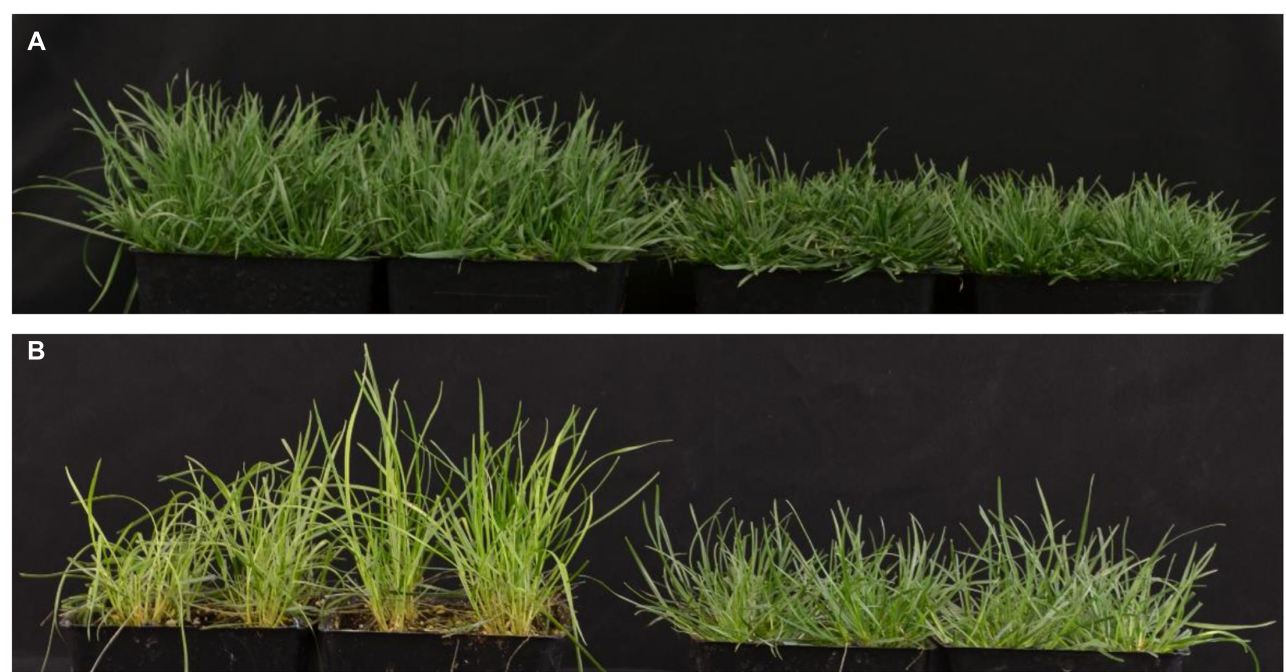

FIGURE 1 | shadow-1 plants exhibit a dual phenotype of dwarfism and shade tolerance. (A) Eight-week-old, wild type (left) and shadow-1 plants (right) grown under full light in the greenhouse. (B) Wild type (left) and shadow-1 plants (right) after 2 weeks under $95 \%$ shade in the greenhouse.

TABLE 1 | Summary of sequencing quality.

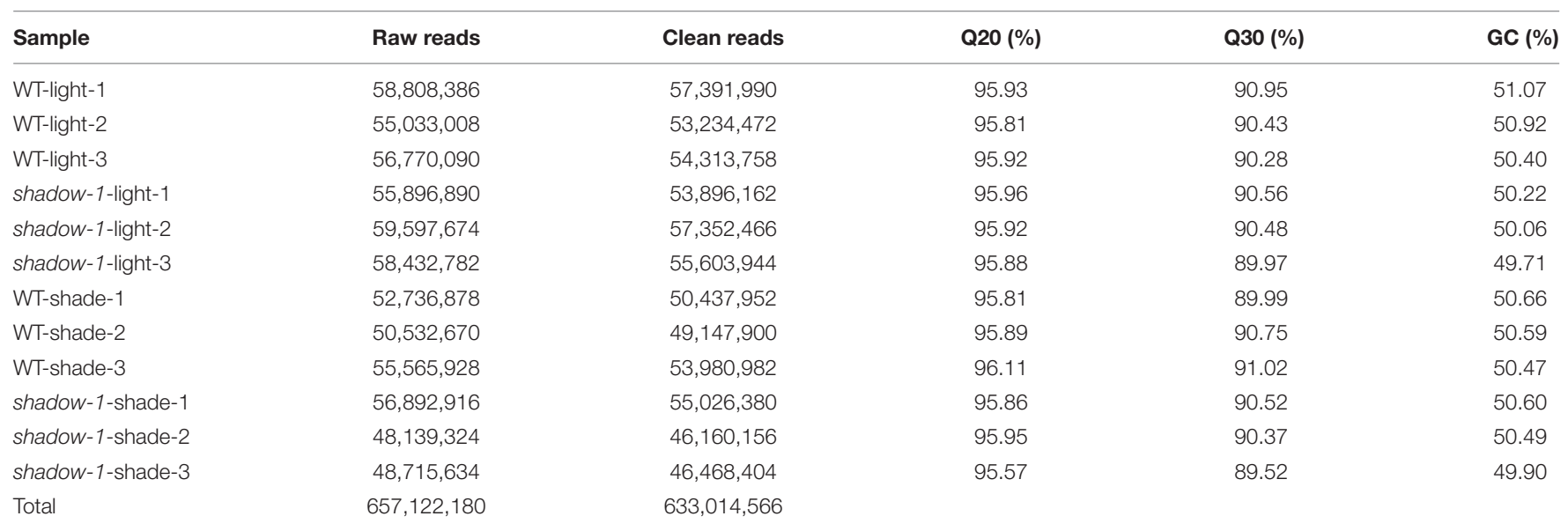

Q20, percentage of bases with a Phred value $>20$; Q30, percentage of bases with a Phred value $>30$.

TABLE 2 | Clean reads were mapped at high percentage to the perennial ryegrass genome.

\begin{tabular}{lcc}
\hline Sample group & Total clean reads & Mapped reads (\%) \\
\hline wild type light & $164,940,220$ & 75.80 \\
shadow-1 light & $166,852,572$ & 75.18 \\
wild type shade & $153,566,834$ & 74.84 \\
shadow-1 shade & $147,654,940$ & 76.64 \\
\hline
\end{tabular}

\section{Differential Gene Expression}

When we examined the number of DEGs for each of these four comparisons (Figure 3), we observed that shade treatment caused more changes in gene expression (i.e., more DEGs) than the mutation(s) in shadow-1 plants under either light or shade conditions. These results demonstrate that shade stress has a larger impact on gene expression than the mutation(s).
There were 4,022 DEGs in shade-treated wild-type plants, compared to those which were grown in the light, with 1,392 up-regulated and 2,630 down-regulated. Similarly, there were 4,067 DEGs (1,374 up-regulated and 2,693 down-regulated) in shade-treated shadow-1 plants compared to those kept under light (Figure 3). 2,668 DEGs (820 up-regulated and 1,848 downregulated) were shared between shade-treated shadow-1 and shade-treated wild-type plants, when each were compared to their light-grown counterparts (Figure $\mathbf{4 A}$ ). It is likely that many of these genes are not involved in the shade tolerance exhibited by shadow-1 plants, but instead are representative of the general shade response of perennial ryegrass.

There were 2,245 DEGs (1,153 up-regulated and 1,092 down-regulated) uncovered in light-grown shadow-1 plants, compared to wild-type and 2,200 DEGs (1,096 up-regulated and 1,104 down-regulated) uncovered in shade-treated shadow1 plants, compared to wild type (Figure 3). There were 


\begin{tabular}{|c|c|c|c|c|c|c|c|c|c|c|c|c|c|}
\hline Replicate & WT-L1 & WT-L2 & WT-L3 & M-L1 & $M-L 2$ & M-L3 & WT-S1 & WT-S2 & WT-S3 & M-S1 & M-S2 & M-S3 & 1 \\
\hline WT-L1 & 1 & 0.999617 & 0.998942 & 0.957664 & 0.961223 & 0.960943 & 0.263007 & 0.263044 & 0.239864 & 0.265505 & 0.266995 & 0.265004 & 0.75 \\
\hline WT-L2 & 0.999617 & 1 & 0.998697 & 0.960227 & 0.963428 & 0.963322 & 0.263825 & 0.263703 & 0.241033 & 0.265704 & 0.267266 & 0.265274 & 0.5 \\
\hline WT-L3 & 0.998942 & 0.998697 & 1 & 0.955528 & 0.959881 & 0.95967 & 0.275667 & 0.275806 & 0.255746 & 0.280335 & 0.281993 & 0.28036 & 0.25 \\
\hline M-L1 & 0.957664 & 0.960227 & 0.955528 & 1 & 0.999226 & 0.999505 & 0.236871 & 0.23665 & 0.220563 & 0.250124 & 0.251723 & 0.250052 & 0 \\
\hline M-L2 & 0.961223 & 0.963428 & 0.959881 & 0.999226 & 1 & 0.999661 & 0.236539 & 0.236709 & 0.219812 & 0.25284 & 0.25432 & 0.252873 & \\
\hline M-L3 & 0.960943 & 0.963322 & 0.95967 & 0.999505 & 0.999661 & 1 & 0.242219 & 0.242019 & 0.22668 & 0.25666 & 0.25832 & 0.256773 & \\
\hline WT-S1 & 0.263007 & 0.263825 & 0.275667 & 0.236871 & 0.236539 & 0.242219 & 1 & 0.99843 & 0.961942 & 0.927166 & 0.93136 & 0.92579 & \\
\hline WT-S2 & 0.263044 & 0.263703 & 0.275806 & 0.23665 & 0.236709 & 0.242019 & 0.99843 & 1 & 0.960912 & 0.935001 & 0.937797 & 0.933631 & \\
\hline WT-S3 & 0.239864 & 0.241033 & 0.255746 & 0.220563 & 0.219812 & 0.22668 & 0.961942 & 0.960912 & 1 & 0.914132 & $\mid 0.918829$ & 0.919555 & \\
\hline M-S1 & 0.265505 & 0.265704 & 0.280335 & 0.250124 & 0.25284 & 0.25666 & 0.927166 & 0.935001 & 0.914132 & 1 & 0.99947 & 0.99938 & \\
\hline M-S2 & 0.266995 & 0.267266 & 0.281993 & 0.251723 & 0.25432 & 0.25832 & 0.93136 & 0.937797 & 0.918829 & 0.99947 & 1 & 0.999137 & \\
\hline M-S3 & 0.265004 & 0.265274 & 0.28036 & 0.250052 & 0.252873 & 0.256773 & 0.92579 & $|0.933631|$ & 0.919555 & 0.99938 & 0.999137 & 1 & \\
\hline
\end{tabular}

FIGURE 2 | Each sample type shows a high degree of consistency between replicates. Comparisons of gene expression between replicates on the $x$-axis and those on the $y$-axis. Pearson correlation coefficient, as well as a color value (whiter-less similar; redder-more similar), are given for each comparison.

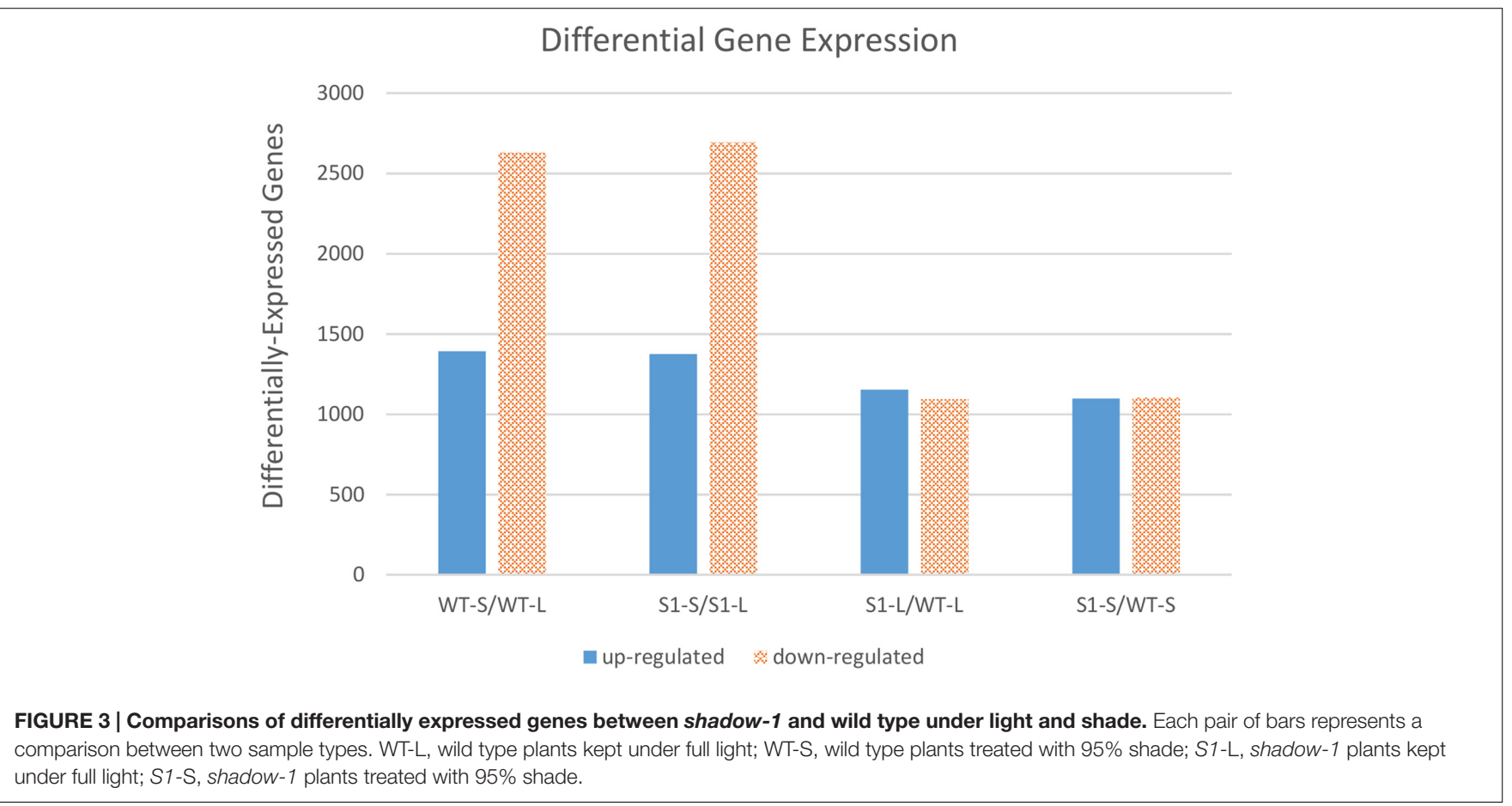

1,240 DEGs (624 up-regulated and 616 down-regulated) shared by shadow-1 plants under light and shade, when each were compared to wild-type under the same conditions. 1,005 DEGs (529 up-regulated, 476 down-regulated) were found only in light-grown shadow-1 (compared to wild type) and 960 DEGs (472 up-regulated, 488 down-regulated) found only in shade-treated shadow-1 (compared to wild type) (Figure 4B). 

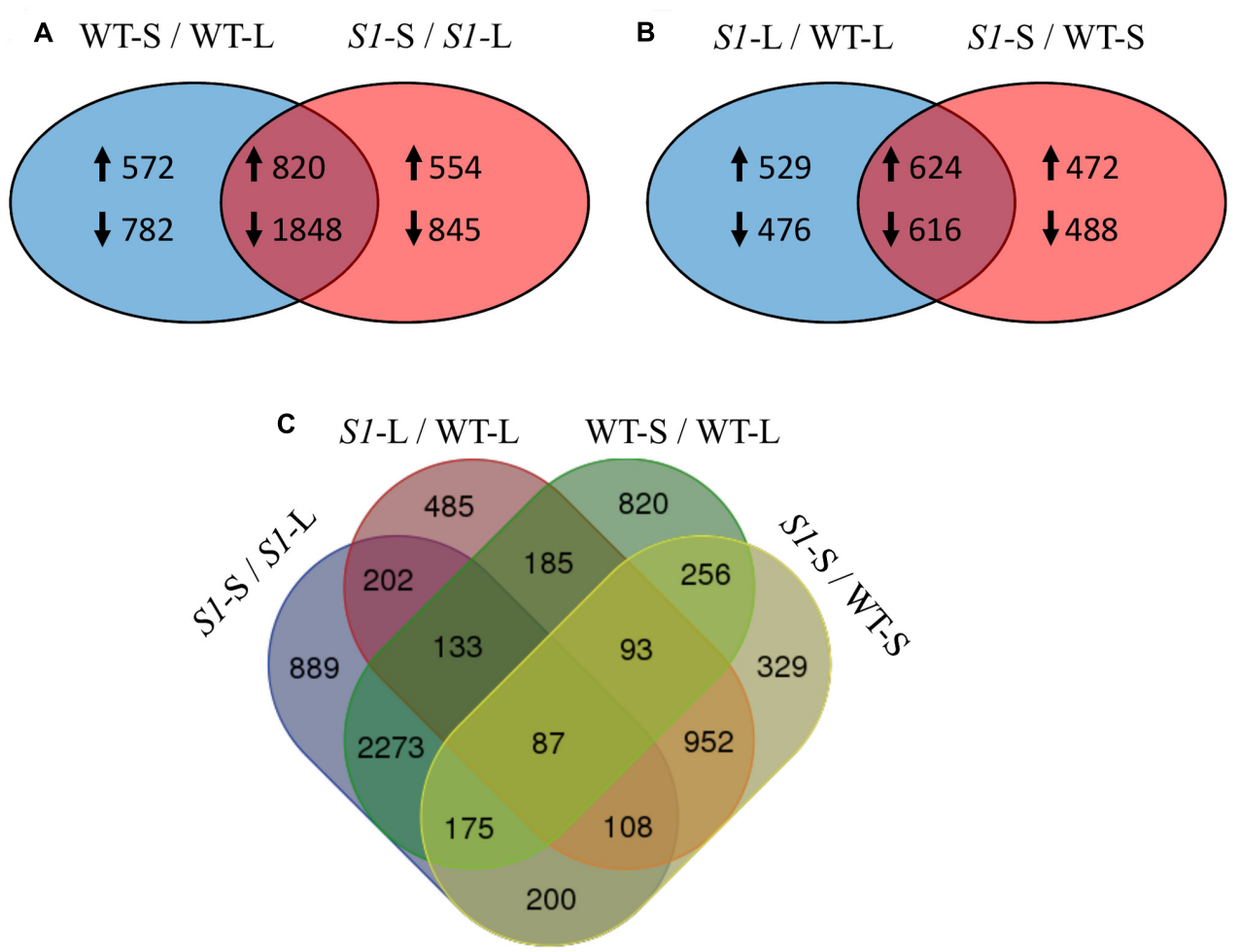

FIGURE 4 | Overlapping of differentially expressed genes across treatments (shade vs full light) and genotypes (wild type vs shadow-1).

(A) Comparison between DEGs identified in wild type following shade treatment (left), to those identified in shadow-1 following shade treatment (right). The overlapping region represents DEGs shared between shadow-1 and wild type following shade treatment. (B) Comparison between DEGs identified in shadow-1 (vs wild type) under light (left) to those identified following shade treatment (right). The overlapping region represents DEGs shared between shadow-1 plants kept under light and those treated with shade, compared to wild type under the same conditions. (A,B) Up-arrows signify up-regulated DEGs and down-arrows signify down-regulated DEGs. (C) Four-way Venn figure including all comparisons from (A) and (B). WT-L, wild type plants kept under full light; WT-S, wild type plants treated with 95\% shade; S1-L, shadow-1 plants kept under full light; S1-S, shadow-1 plants treated with 95\% shade.

We also compared differential gene expression between lightgrown shadow-1, shade-treated shadow-1, light-grown wildtype, and shade-treated wild type, in a four-way comparison (Figure 4C). This four-way comparison exposed 329 DEGs that were unique to shadow-1 when compared to wild-type under shade conditions, and 485 DEGs that were unique to shadow-1 if compared to wild type under light conditions. There were also 820 DEGs that were unique to shade-treated wild-type plants (compared to wild type under light), and 889 DEGs that were unique to shade-treated shadow-1 plants (compared to shadow-1 under light). There were 87 DEGs that had differential expression in shadow-1 and wild type under both light and shade.

In order to explore the function of DEGs identified in shadow-1 plants (compared to wild type) under both light and shade conditions, we performed GO enrichment analysis. The enriched GO distributions were similar for shadow-1 plants under light and under shade (compared to wild type under the same conditions), however, there were a few notable differences. We examined some of the genes from the GO groups that showed differences and queried the associated proteins against the NCBI NR protein database. DEGs involved in "biological adhesion" and "receptor activity" for shadow-1 plants kept under light were absent in shadow-1 plants treated with shade (Figure 5).
The "biological adhesion" group included a gene that coded for ERECTA, a receptor-like kinase, and was up-regulated $(9.85 \times)$ in shadow-1 plants compared to wild type under light. The "receptor activity" group included a gene that coded for PhyA, a light receptor, was also up-regulated $(7.24 \times)$ in shadow-1 plants under light. For another GO group, “extracellular region part," shadow-1 plants under light had only down-regulated DEGs, while shadetreated shadow-1 plants had both up- and down-regulated DEGs (Figure 5). One gene within this group coded for cytokinin oxygenase, which degrades bioactive cytokinin, and was upregulated $(11.38 \times)$ in shadow-1 plants under shade compared to wild type, but was not differentially regulated in shadow-1 under light.

\section{DEGs in the GA Pathway}

Previously, we showed that the dwarfism and shade-tolerance phenotypes displayed in shadow-1 might be caused by changes in GA concentration ( $\mathrm{Li}$ et al., 2016). The genes responsible for GA biosynthesis are poorly annotated in perennial ryegrass, but are well characterized in bread wheat (Triticum aestivum), a close relative of perennial ryegrass. To uncover DEGs within the GA biosynthesis pathway, we selected the protein sequences for enzymes catalyzing key steps of GA biosynthesis in bread 

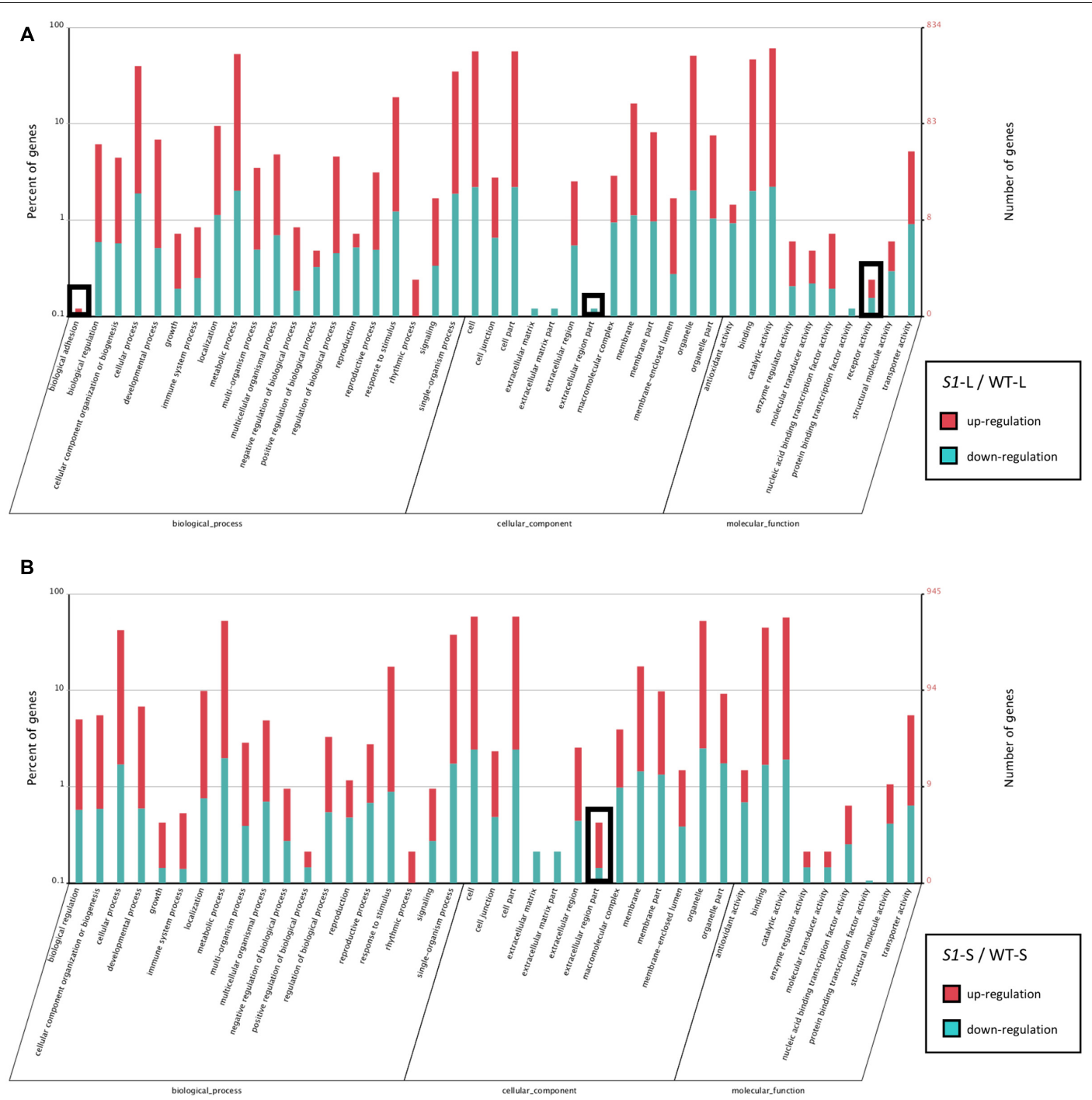

FIGURE 5 | Functional gene classification of DEGs. (A) Gene Ontology (GO) distribution of DEGs identified in shadow-1 plants compared to wild type kept under full light. (B) GO distribution of DEGs identified in shadow-1 plants compared to wild type after shade treatment. Black boxes highlight differences between specific terms in (A) and (B). S1-L, shadow-1 plants kept under full light; WT-L, wild type plants kept under full light; S1-S, shadow-1 plants treated with 95\% shade; WT-S, wild type plants treated with $95 \%$ shade.

wheat and aligned them to the translated perennial ryegrass reference genome (Supplementary File 2). As shown in Figure 6, putative GA biosynthesis genes were down-regulated in shadow-1 plants (compared to wild type) under both light and shade conditions. Under light, the GA biosynthesis genes, CPS, KS, $K O$, and $K A O$, were down-regulated to $24.4-84.7 \%$ of the levels of wild-type plants. Under shade conditions, these genes were also down-regulated to $17.4-61.4 \%$ of the wild type plants. The downstream GA biosynthesis splits into two pathways, one for $\mathrm{GA}_{1}$ and another for $\mathrm{GA}_{4}$. Both pathways are catalyzed by GA20ox followed by GA3ox, which are responsible for key steps in GA biosynthesis. Expression of GA20ox was reduced in shadow-1 plants kept under light, to $39.0 \%$ of the expression in wild type. Under the shade conditions, the expression of GA20ox in shadow-1 plants was reduced to $3.3 \%$ when compared to the wild type control. We were unable to uncover putative homologs of GA3ox through annotation.

We next examined one GA response gene, lipid transfer protein 3 (LTP3), which is up-regulated by GA (De Lucas et al., 2008). LTP3 was down-regulated to $41.5 \%$ in shadow- 1 plants if compared to its expression in wild type under shade conditions (Figure 7). This gene was also down-regulated in shadow-1 plants when comparing to the wild-type under nature light (data not shown).

\section{Verification of Differentially Expressed Genes via qRT-PCR}

We verified the accuracy of our transcriptome data by selecting three genes (KS, KAO, and GA20ox) for qRT-PCR analysis, using 


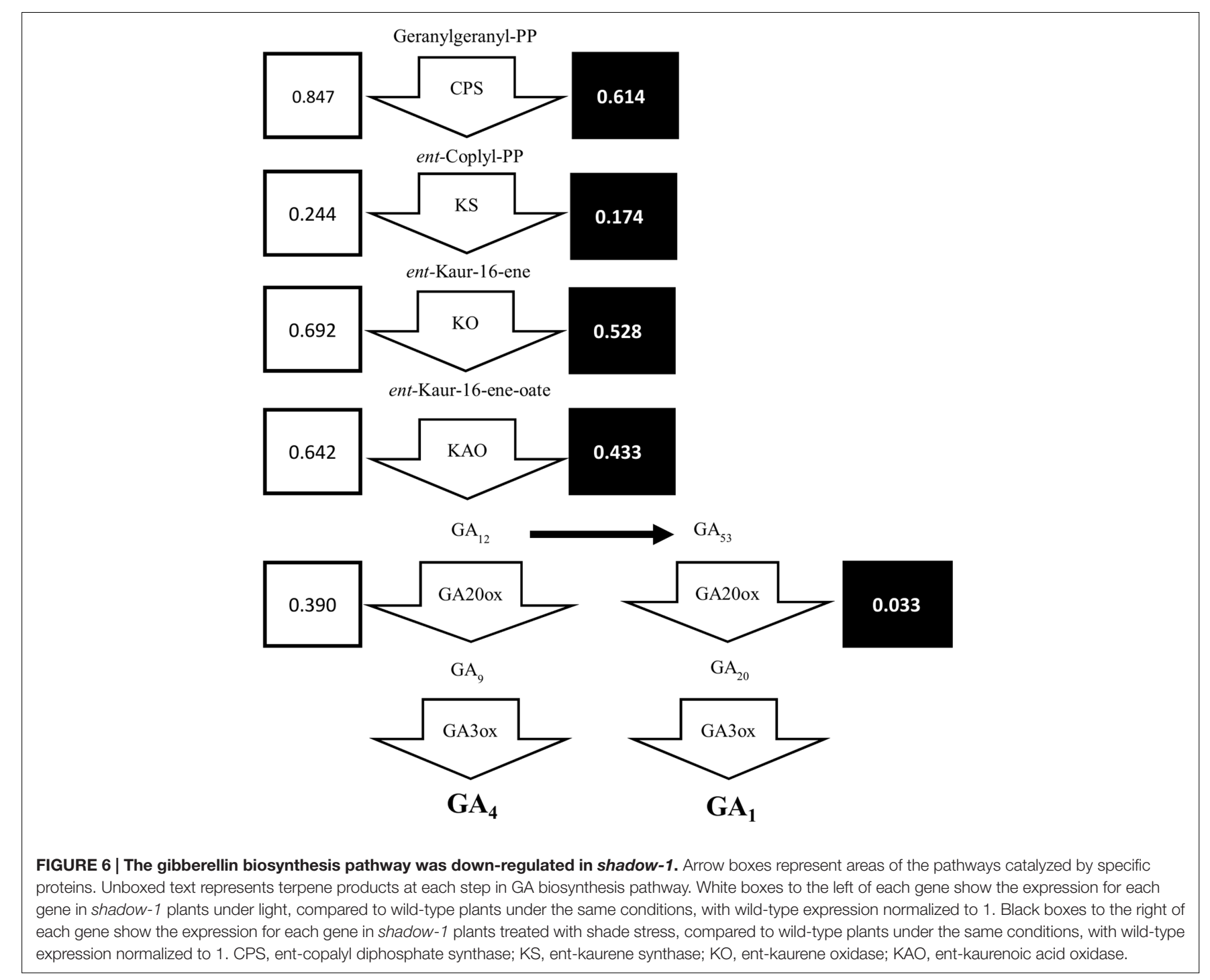

mRNA extracted from shade-treated wild-type and shadow1 plants. The results of qRT-PCR analysis showed similar expression patterns to those obtained from our transcriptome analysis (Figure 8). Transcriptome analysis demonstrated that, in shade-treated shadow-1, KS expression was reduced to $17.4 \%$ of its expression in wild type, while qRT-PCR showed downregulation to $14.8 \%$ of wild-type expression. Under the same conditions, transcriptome analysis showed that KAO was downregulated in shadow-1 to $43.3 \%$ of its wild-type expression, while qRT-PCR showed down-regulation to $49.7 \%$. For shadetreated shadow-1, GA20ox was down-regulated to $3.3 \%$ of its expression in wild type. $3.3 \%$ represents a total of only 0.03 mapped reads (RPKM) over millions of sequencing reactions, which is in the barely detectable level. Consistently, we could not detect expression of this gene for these plants with our qRT-PCR analysis. In summary, the expression patterns detected with the transcriptome and $\mathrm{qPCR}$ analyses are consistent in general, demonstrating that the transcriptome data from Illumina sequencing analysis are reliable.

\section{DISCUSSION}

In this study, we analyzed the transcriptomes of the shadetolerant perennial ryegrass mutant shadow-1 kept under light and shade, to explore the mechanisms behind both dwarfism and shade-tolerance in these plants. We discovered that, in the light, there were noteworthy differences in gene expression between the shadow-1 mutant and wild type, in the form of 2,245 DEGs. There were similar differences in gene expression between these two genotypes after both were subjected to shade stress (2,200 DEGs). When we compared DEGs across genotypes and across treatments, there were 485 DEGs that were unique to shadow-1 (compared to wild type) under light, and there were 329 DEGs that were unique to shadow-1 (compared to wild type) under shade. Additionally, we uncovered 87 DEGs which were differentially expressed in shadow-1 (compared to wild type) under light and shade, and were also differentially expressed in wild type under shade (compared to wild type under light) and shadow-1 under shade (compared to shadow-1 


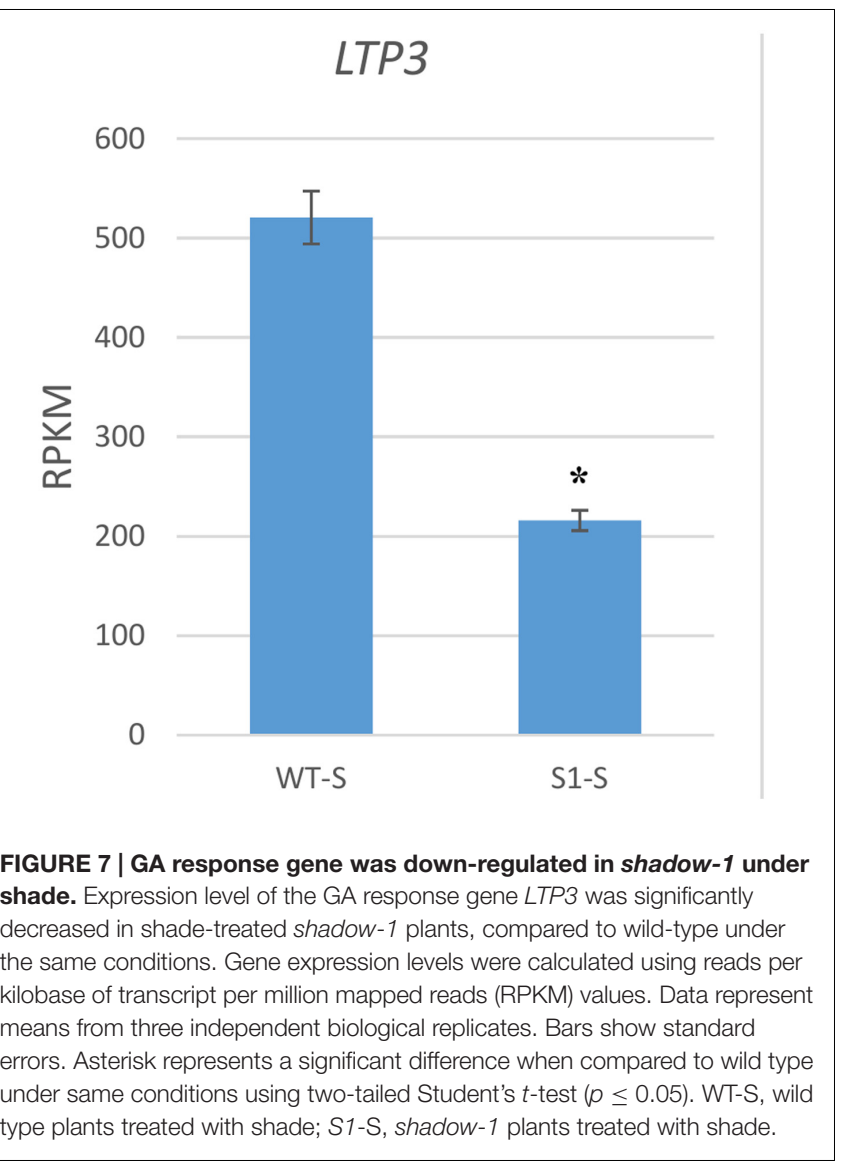

under light). Furthermore, we have observed an overall downregulation of GA biosynthesis genes in shadow-1 plants compared to wild type, under both light and shade conditions, most notably GA20ox, which was down-regulated to $3.3 \%$ in shadow-1 plants under shade conditions. These data provide additional support for our hypothesis ( $\mathrm{Li}$ et al., 2016) that GA plays a key role in both dwarfism and shade tolerance, as shown by shadow-1 plants.

Our results have shown that key GA biosynthesis genes, as well as one GA response gene, were down-regulated in the shadow-1 transcriptome compared to wild type, under both light and shade conditions. We also checked the expression of GID1, the main receptor of GA, and found there was no difference in expression at mRNA level between shadow-1 and wild type under either light or shade conditions. Evidence pointing toward decreased GA biosynthesis in shadow-1 plants is consistent with our previous report that both dwarfism and shade tolerance in shadow-1 plants can be abolished through exogenous application of gibberellic acid (GA3; Li et al., 2016). We have also previously shown that interruption of GA biosynthesis, through the application of trinexapac-ethyl (TE) to wild-type plants, is sufficient to cause dwarfism and shade tolerance in these plants. TE acts by disrupting the latter steps of GA biosynthesis, such as those controlled by GA20ox (Hedden and Thomas, 2012), which has now been shown to be down-regulated in shadow-1 plants under both light and shade conditions. Our data suggest that dwarfism and shade tolerance are connected in shadow-1 through the activity of GA. If this is the case, it is likely that dwarfism, through the mechanism of reduced leaf elongation, provides tolerance to shade stress.

Through Illumina sequencing, we generated millions of clean reads, representing gigabytes of sequencing data, which were efficiently mapped to a reference genome. There was high consistency between the biological replicates used for RNA sequencing ( $r \geq 96 \%$ ), demonstrating the reliability of the data produced. We were also able to confirm the accuracy of our transcriptome analysis via qRT-PCR analysis. Together, these data demonstrate the excellent reproducibility of the results. The transcriptome data acquired from shadow-1 plants under light and shade conditions are valuable resources for exploring genetic mechanisms underlying dwarfism and shade tolerance.

We were unable to pinpoint the exact gene(s) whose mutation was responsible for the mutant phenotypes exhibited by shadow-1 plants. However, these mutant genes cause downstream changes in gene expression that are observable through our transcriptome analysis. If the dwarf and shade-tolerant phenotypes exhibited by shadow-1 plants are caused by the same mutation(s), the DEGs resulting from the mutation(s) should be shared by shadow-1 under light and shadow-1 under shade (compared to wild type under the same conditions). Because of the down regulation of the GA biosynthesis genes observed in the shadow-1 mutant plants, one possibility is that a key gene such as a transcription factor gene involved in regulating the overall GA biosynthetic pathway may be knocked out. Further characterization of the mutant plants is needed to address these questions.

As was shown in our GO enrichment analysis, there were differences in the gene expression of shadow-1 plants (compared to wild type), depending on whether plants were kept under light or were treated with shade, which provides insight into potential differences between dwarfism and shade tolerance for these plants. We identified three of these genes, coding for ERECTA (up-regulated in shadow-1 in light), PhyA (up-regulated in shadow-1 in light), and cytokinin deoxygenase (up-regulated in shadow-1 in shade). ERECTA and cytokinin deoxygenase are both related to cell division/proliferation (Riou-Khamlichi et al., 1999; Shpak et al., 2004), which could have an impact on dwarfism and shade tolerance by influencing leaf elongation. Leaf elongation is associated with GA response, especially under low-light conditions (Tan and Qian, 2003; Xu et al., 2016), making it likely that the expression of these two genes have some connection to downstream GA signaling. Additionally, phytochrome is indirectly involved in the GA response pathway through the activity of PIFs, which act as transcription factors and have been implicated in the GA-mediated light response (Alabadí et al., 2008; Lorrain et al., 2008; Shin et al., 2009). It would be interesting to further dissect the role of these genes in shade responses of turf grasses.

The shadow-1 mutant line may serve as a good model plant for the study of mechanisms leading to dwarfism and shade tolerance in plants. Both of these traits have utility for plant breeders, in areas ranging from agricultural to ornamental (Wilkins, 1991). Dwarf plants can have increased crop yields and could 

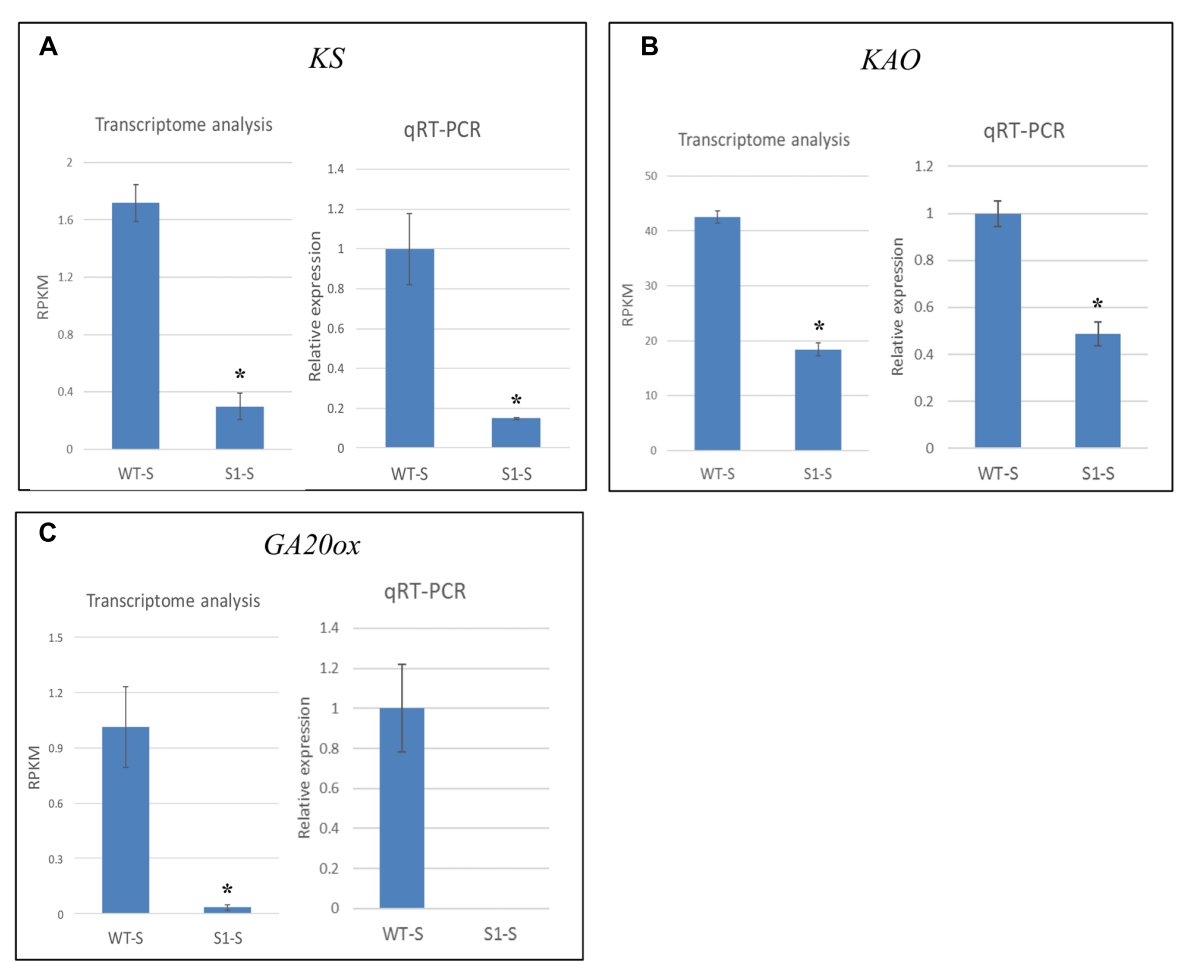

FIGURE 8 | qRT-PCR data verified the accuracy of transcriptome analysis. Expression levels of KS (A), KAO (B), and GA20ox (C) were identified through transcriptome and qRT-PCR analyses. For transcriptome analysis, gene expression levels were calculated using reads per kilobase of transcript per million mapped reads (RPKM) values. For qRT-PCR, the gene expression levels in each sample were normalized using the expression level of the internal control, $L p G A P D H$, in the same sample, and wild-type expression levels were normalized to 1. The data presented are the means from three independent biological replicates. Bars show standard errors. Asterisk represents a significant difference when compared to wild type under same conditions using two-tailed Student's $t$-test ( $p \leq 0.05)$. WT-S, wild type plants treated with shade; S1-S, shadow-1 plants treated with shade.

have reduced requirements for nutrients (Monna et al., 2002). Shade-tolerant plants are able to thrive in environments that are traditionally unconducive to healthy plant growth, such as under tree canopies or in dense urban areas (Jiang et al., 2004). Our transcriptome analysis suggests that, in shadow-1 plants, the genetic mechanism of both dwarfism and shade tolerance is the down-regulation of genes across the GA biosynthesis pathway. This information could be valuable to turf geneticists and breeders who are interested in developing new cultivars that have either, or both, of these traits.

\section{AUTHOR CONTRIBUTIONS}

WL and LK-G are equally contributed and did most of the work for mutant characterization. XG and XW were responsible for mapping of transcriptome data. WL, LK-G, XG, XW, and RE-T were responsible for analysis of DEGs. RE-T and HY were also involved greenhouse and field evaluation of the mutant. CT was involved in gamma-ray irradiation and producing M2 seeds and initial screening of the mutant. JI, KG, and RM provided advices on characterization of the mutant and manuscript editing. JW provided advices on transcriptome data analysis and manuscript editing. YL and TG designed experiments for the mutant characterization and transcriptome analysis. WL, LK-G, and YL were involved in manuscript writing and editing.

\section{FUNDING}

This project is financially supported by the Storrs Agricultural Experiment Station to YL.

\section{ACKNOWLEDGMENTS}

We thank the personnel at the University of Massachusetts for their kind assistance in gamma-ray irradiation of seed materials. We also thank Mr. Steve Olsen and his crew at the University of Connecticut, Plant Science Research and Education Facility for their help in growing and maintaining mutant plant populations. We would like to acknowledge UConn's Computational Biology Core for hardware, software, and technical support.

\section{SUPPLEMENTARY MATERIAL}

The Supplementary Material for this article can be found online at: http://journal.frontiersin.org/article/10.3389/fpls.2017.00868/ full\#supplementary-material 


\section{REFERENCES}

Alabadí, D., Gallego-Bartolomé, J., Orlando, L., García-Cárcel, L., Rubio, V., Martínez, C., et al. (2008). Gibberellins modulate light signaling pathways to prevent Arabidopsis seedling de-etiolation in darkness. Plant J. 53, 324-335. doi: 10.1111/j.1365-313X.2007.03346.x

Byrne, S. L., Nagy, I., Pfeifer, M., Armstead, I., Swain, S., Studer, B., et al. (2015). A synteny-based draft genome sequence of the forage grass Lolium perenne. Plant J. 84, 816-826. doi: 10.1111/tpj.13037

Chen, J., Thammina, C., Li, W., Yu, H., Yer, H., El-Tanbouly, R., et al. (2016). Isolation of prostrate turfgrass mutants via screening of dwarf phenotype and characterization of a perennial ryegrass prostrate mutant. Hortic. Res. 3, 16003. doi: 10.1038/hortres. 2016.3

Chen, S., Wang, X., Zhang, L., Lin, S., Liu, D., Wang, Q., et al. (2016). Identification and characterization of tomato gibberellin 2-oxidases (GA2oxs) and effects of fruit-specific SIGA2ox1 overexpression on fruit and seed growth and development. Hortic. Res. 3, 16059. doi: 10.1038/hortres.2016.59

Colebrook, E. H., Thomas, S. G., Phillips, A. L., and Hedden, P. (2014). The role of gibberellin signalling in plant responses to abiotic stress. J. Exp. Biol. 217, 67-75. doi: $10.1242 /$ jeb.089938

Conesa, A., and Götz, S. (2008). Blast2GO: a comprehensive suite for functional analysis in plant genomics. Int. J. Plant Genomics 2008:619832. doi: 10.1155/ 2008/619832

De Lucas, M., Daviere, J.-M., Rodriguez-Falcon, M., Pontin, M., Iglesias-Pedraz, J. M., Lorrain, S., et al. (2008). A molecular framework for light and gibberellin control of cell elongation. Nature 451, 480-484. doi: 10.1038/nature06520

Feng, S., Martinez, C., Gusmaroli, G., Wang, Y., Zhou, J., Wang, F., et al. (2008). Coordinated regulation of Arabidopsis thaliana development by light and gibberellins. Nature 451, 475-479. doi: 10.1038/nature06448

Franklin, K. A., and Whitelam, G. C. (2005). Phytochromes and shade-avoidance responses in plants. Ann. Bot. 96, 169-175. doi: 10.1093/aob/mci165

Gardner, D., and Taylor, J. (2002). Change over time in quality and cover of various turfgrass species and cultivars maintained in shade. HortTechnology 12, 465-469.

Grinberg, N. F., Lovatt, A., Hegarty, M., Lovatt, A., Skøt, K. P., Kelly, R., et al. (2016). Implementation of genomic prediction in Lolium perenne (L.) breeding populations. Front. Plant Sci. 7:133. doi: 10.3389/fpls.2016.00133

Guindon, S., Dufayard, J.-F., Lefort, V., Anisimova, M., Hordijk, W., and Gascuel, O. (2010). New algorithms and methods to estimate maximumlikelihood phylogenies: assessing the performance of PhyML 3.0. Syst. Biol. 59, 307-321. doi: 10.1093/sysbio/syq010

Hedden, P., and Phillips, A. L. (2000). Gibberellin metabolism: new insights revealed by the genes. Trends Plant Sci. 5, 523-530. doi: 10.1016/S13601385(00)01790-8

Hedden, P., and Thomas, S. G. (2012). Gibberellin biosynthesis and its regulation. Biochem. J. 444, 11-25. doi: 10.1042/BJ20120245

Jiang, Y., Duncan, R. R., and Carrow, R. N. (2004). Assessment of low light tolerance of seashore paspalum and bermudagrass. Crop Sci. 44, 587-594. doi: $10.2135 /$ cropsci2004.5870

Jiang, Y., and Huang, B. (2001). Physiological responses to heat stress alone or in combination with drought: a comparison between tall fescue and perennial ryegrass. HortScience 36, 682-686.

Kebrom, T. H., Burson, B. L., and Finlayson, S. A. (2006). Phytochrome B represses Teosinte Branched1 expression and induces sorghum axillary bud outgrowth in response to light signals. Plant Physiol. 140, 1109-1117. doi: 10.1104/pp.105. 074856

Kim, D., Pertea, G., Trapnell, C., Pimentel, H., Kelley, R., and Salzberg, S. L. (2013). TopHat2: accurate alignment of transcriptomes in the presence of insertions, deletions and gene fusions. Genome Biol. 14:R36. doi: 10.1186/gb-2013-14-4r36

Kovi, M. R., Abdelhalim, M., Kunapareddy, A., Ergon, Å., Tronsmo, A. M., Brurberg, M. B., et al. (2016). Global transcriptome changes in perennial ryegrass during early infection by pink snow mould. Sci. Rep. 6:28702. doi: $10.1038 /$ srep28702

Kozuka, T., Horiguchi, G., Kim, G.-T., Ohgishi, M., Sakai, T., and Tsukaya, H. (2005). The different growth responses of the Arabidopsis thaliana leaf blade and the petiole during shade avoidance are regulated by photoreceptors and sugar. Plant Cell Physiol. 46, 213-223. doi: 10.1093/pcp/pci016
Larkin, M. A., Blackshields, G., Brown, N., Chenna, R., McGettigan, P. A., McWilliam, H., et al. (2007). Clustal W and Clustal X version 2.0. Bioinformatics 23, 2947-2948. doi: 10.1093/bioinformatics/btm404

Li, W., Katin-Grazzini, L., Krishnan, S., Thammina, C., El-Tanbouly, R., Yer, H., et al. (2016). A novel two-step method for screening shade tolerant mutant plants via dwarfism. Front. Plant Sci. 7:1495. doi: 10.3389/fpls.2016.01495

Lorrain, S., Allen, T., Duek, P. D., Whitelam, G. C., and Fankhauser, C. (2008). Phytochrome-mediated inhibition of shade avoidance involves degradation of growth-promoting bHLH transcription factors. Plant J. 53, 312-323. doi: 10.1111/j.1365-313X.2007.03341.x

Monna, L., Kitazawa, N., Yoshino, R., Suzuki, J., Masuda, H., Maehara, Y., et al. (2002). Positional cloning of rice semidwarfing gene, sd-1: rice "green revolution gene" encodes a mutant enzyme involved in gibberellin synthesis. DNA Res. 9, 11-17. doi: 10.1093/dnares/9.1.11

Nozue, K., Tat, A. V., Kumar Devisetty, U., Robinson, M., Mumbach, M. R., Ichihashi, Y., et al. (2015). Shade avoidance components and pathways in adult plants revealed by phenotypic profiling. PLoS Genet. 11:e1004953. doi: 10.1371/ journal.pgen.1004953

Page, E., Tollenaar, M., Lee, E., Lukens, L., and Swanton, C. (2010). Shade avoidance: an integral component of crop-weed competition. Weed Res. 50, 281-288. doi: 10.1111/j.1365-3180.2010.00781.x

Park, E., Park, J., Kim, J., Nagatani, A., Lagarias, J. C., and Choi, G. (2012). Phytochrome B inhibits binding of phytochrome-interacting factors to their target promoters. Plant J. 72, 537-546. doi: 10.1111/j.1365-313X.2012.05114.x

Petersen, K., Didion, T., Andersen, C. H., and Nielsen, K. K. (2004). MADSbox genes from perennial ryegrass differentially expressed during transition from vegetative to reproductive growth. J. Plant Physiol. 161, 439-447. doi: 10.1078/0176-1617-01212

Pons, T., and Poorter, H. (2014). The effect of irradiance on the carbon balance and tissue characteristics of five herbaceous species differing in shade-tolerance. Front. Plant Sci. 5:12. doi: 10.3389/fpls.2014.00012

Procko, C., Crenshaw, C. M., Ljung, K., Noel, J. P., and Chory, J. (2014). Cotyledongenerated auxin is required for shade-induced hypocotyl growth in Brassica rapa. Plant Physiol. 165, 1285-1301. doi: 10.1104/pp.114.241844

Riou-Khamlichi, C., Huntley, R., Jacqmard, A., and Murray, J. A. (1999). Cytokinin activation of Arabidopsis cell division through a D-type cyclin. Science 283, 1541-1544. doi: 10.1126/science.283.5407.1541

Sasidharan, R., Chinnappa, C., Staal, M., Elzenga, J. T. M., Yokoyama, R., Nishitani, K., et al. (2010). Light quality-mediated petiole elongation in Arabidopsis during shade avoidance involves cell wall modification by xyloglucan endotransglucosylase/hydrolases. Plant Physiol. 154, 978-990. doi: $10.1104 /$ pp.110.162057

Shin, J., Kim, K., Kang, H., Zulfugarov, I. S., Bae, G., Lee, C.-H., et al. (2009). Phytochromes promote seedling light responses by inhibiting four negativelyacting phytochrome-interacting factors. Proc. Natl. Acad. Sci. U.S.A. 106 7660-7665. doi: 10.1073/pnas.0812219106

Shpak, E. D., Berthiaume, C. T., Hill, E. J., and Torii, K. U. (2004). Synergistic interaction of three ERECTA-family receptor-like kinases controls Arabidopsis organ growth and flower development by promoting cell proliferation. Development 131, 1491-1501. doi: 10.1242/dev.01028

Steel, R. G., Torrie, J. H., and Dickey, J. H. (1997). Principles and Procedures of Statistics a Biometrical Approach, 3rd Edn. New York, NY: McGraw Hill Book Co.

Sun, T.-P. (2008). Gibberellin metabolism, perception and signaling pathways in Arabidopsis. Arabidopsis Book 6:e0103. doi: 10.1199/tab.0103

Tan, Z., and Qian, Y. (2003). Light intensity affects gibberellic acid content in Kentucky bluegrass. HortScience 38, 113-116.

Tegg, R., and Lane, P. (2004). A comparison of the performance and growth of a range of turfgrass species under shade. Anim. Prod. Sci. 44, 353-358. doi: 10.1071/EA02159

Whipple, C. J., Kebrom, T. H., Weber, A. L., Yang, F., Hall, D., Meeley, R., et al. (2011). Grassy tillers1 promotes apical dominance in maize and responds to shade signals in the grasses. Proc. Natl. Acad. Sci. U.S.A. 108, E506-E512. doi: 10.1073/pnas.1102819108

Wilkins, P. W. (1991). Breeding perennial ryegrass for agriculture. Euphytica 52 201-214. doi: 10.1007/bf00029397

Xu, Q., Krishnan, S., Merewitz, E., Xu, J., and Huang, B. (2016). Gibberellinregulation and genetic variations in leaf elongation for tall fescue in association 
with differential gene expression controlling cell expansion. Sci. Rep. 6:30258. doi: $10.1038 /$ srep30258

Yamaguchi, S. (2008). Gibberellin metabolism and its regulation. Annu. Rev. Plant Biol. 59, 225-251. doi: 10.1146/annurev.arplant.59.032607.092804

Conflict of Interest Statement: The authors declare that the research was conducted in the absence of any commercial or financial relationships that could be construed as a potential conflict of interest.
Copyright $\odot 2017$ Li, Katin-Grazzini, Gu, Wang, El-Tanbouly, Yer, Thammina, Inguagiato, Guillard, McAvoy, Wegrzyn, Gu and Li. This is an open-access article distributed under the terms of the Creative Commons Attribution License (CC BY). The use, distribution or reproduction in other forums is permitted, provided the original author(s) or licensor are credited and that the original publication in this journal is cited, in accordance with accepted academic practice. No use, distribution or reproduction is permitted which does not comply with these terms. 